\title{
Dennis Gabor - Winner of the 1971 Physics Nobel Prize
}

\section{E. Ingelstam, stockholm}

The 1971 Nobel Prize in Physics was awarded to Dennis Gabor for 'his invention and development of the holographic method'. To start with, a short explanation of this principle may be in order. In imaging processes where light falls on a so-called square-law detector (one responding to the intensity of light or the square of the amplitudes of the light waves) half the information, namely the phase of the optical waves, is lost. This is true for photographic emulsions, photosensitive layers in phototubes, TV camera tubes etc.

In order to obtain the phase, it is necessary to have a reference phase. This is the case in interferometry where two wave fields are superimposed on each other so that one becomes the reference wave of the other. The novelty of Gabor's invention is the use of this principle for twostep imaging. In the first step a wave reflected on to the object whose image is required (or passing through it if it is transparent) falls on a photographic plate which, at the same time, is illuminated by another wave from the same source but not touching the object (the reference wave). In general a very complicated interference pattern is produced on the photographic emulsion. The more details of the object at various distances and in different directions from the plate are involved, the more complex will be this pattern. Gabor called the plate a 'hologram' (from holos $=$ whole, entire) because it contains the whole information of the object, not only that relating to amplitude but also phase.

Once exposed, the hologram is available at any time for the second step of the imaging process. If a new reference wave falls on it, preferably at the same angle as before, the hologram pattern will act as an optical grating or as a myriad of Fresnel zones diffracting the wave so that the former wavefield from the object is reconstructed. In general two images of the object are formed, one real and one virtual, the latter being a true three-dimensional reproduction.
Gabor originally thought of using the principle to make an electron microscope image in two steps: first to record the field of an object as a hologram using electron rays, and then to reconstruct this with visible light to produce a high-resolution three-dimensional image. Suitable electron sources were, however, not available and, for technical reasons, the idea could not be tested. But by successful experiments with light Gabor was able to show that the principle was correct. In three papers, from 1948 to 1951, he gave an exact analysis of the method, and his equations contain all requisite information even for present-day uses.

The break-through of holography came with the arrival of lasers as light sources capable of generating coherent wave trains of such length as to make possible the reconstruction of three-dimensional images of considerable depth. Emmett Leith and his group at Michigan University, USA, and many other institutes after them brought about very rapid technical development and numerous scientific achievements.

Holography enables the position of each point of the object to be determined to a fraction of a wavelength thanks to the phase in the wave field. Thus the hologram has rather unexpectedly enriched optical measurement techniques and, in particular, made possible interferometric measurements on many objects. The changing shape of an object at different times, e.g. in vibration, can be stored in one and the same hologram by exposing the plate several times. When the different stored wave fields are reconstructed simultaneously they interfere with each other and the image of the object is covered with interference lines, which, directly in wavelengths, correspond to the changes of shape between exposures. Very rapid sequences of events, even in plasma physics, are amenable to analysis through hologram exposures made with short light flashes from modern pulsed lasers. A good survey of present-day techniques and applic-

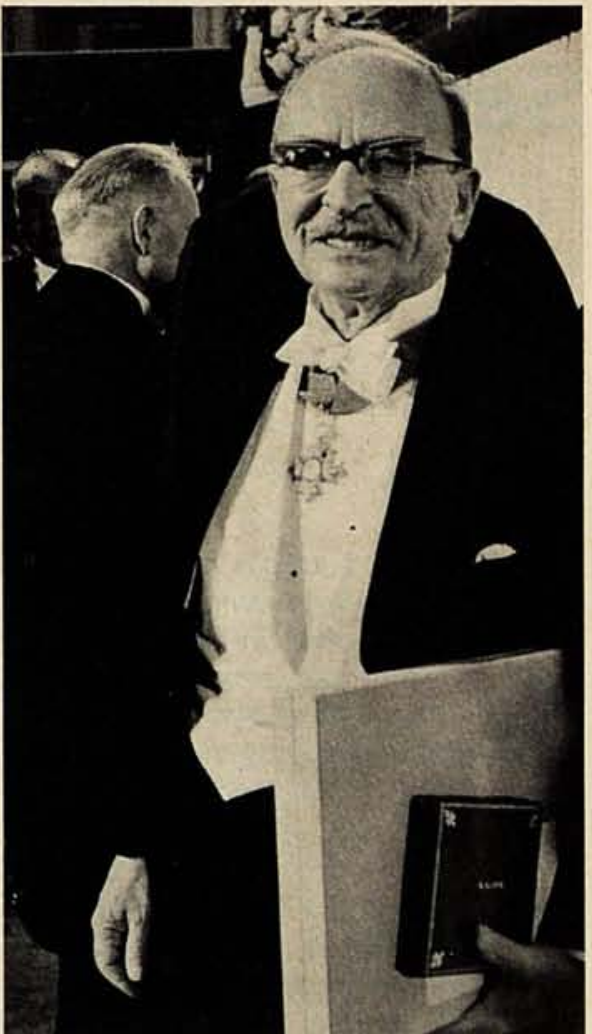

ations will be found in the book by Collier, Burckhardt and Lin : Optical Holography (Academic Press, 1971).

Gabor is really an inventor and, in this respect, accords well with the original idea of Alfred Nobel that the prizes should honour inventions. He has also treated important problems in information theory and now, as Emeritus Professor of Applied Physics at Imperial College, London, and also at the CBS Laboratories in Stanford, USA he continues to add to the development of the field by assiduously contributing ideas for solving practical and theoretical problems.

Dennis Gabor's interest in futurology and social questions is also well known. The last day of his stay in Stockholm after the distribution of the prizes he spent in discussions of the peculiarities and problems of welfarestate society. He is very outspoken in his personal views on such questions as is shown in his book 'Innovations, Scientific, Technological and Social' (Oxford University Press, 1970). He calls for better matching between advanced technology and backward social institutions and is excited about ideas of international citizenship and the education of peace specialists. One has the feeling that NASA may not be very fond of him. Although he appreciates the collective effort put into the Apollo projects he questions their motivation and states that such an effort would deserve much higher priority if dedicated to large-scale social projects, provided the same unity of purpose could be achieved. 